\title{
STM measurements on the InAs(110) surface directly compared with surface electronic structure calculations
}

\author{
Jan Klijn, ${ }^{1}$ Lilli Sacharow, ${ }^{1}$ Christian Meyer, ${ }^{1}$ Stefan Blügel, ${ }^{2}$ Markus Morgenstern, ${ }^{1, *}$ and Roland Wiesendanger ${ }^{1}$ \\ ${ }^{1}$ Institute of Applied Physics, Hamburg University, D-20355 Hamburg, Germany \\ ${ }^{2}$ Institut für Festkörperforschung, Forschungszentrum Jülich, D-52425 Jülich, Germany
}

(Received 16 May 2003; revised manuscript received 20 August 2003; published 24 November 2003)

\begin{abstract}
$A b$ initio density-functional-theory-local-density-approximation electronic structure calculations are performed for the InAs(110) surface and compared with scanning tunnel microscopy (STM) measurements using the Tersoff-Hamann model. In both, calculations and measurements, we see the same atomic features. At negative and small positive energies, the local density of states is concentrated around the As atom, while at higher positive energies it is centered above the In atom, because of the appearance of the In dangling bond. Moreover, we describe two types of irregular STM images on the InAs(110) surface. First, we measure $d I / d V$ images exhibiting atomic resolution at voltages within the band gap, which, however, still can be understood within the Tersoff-Hamann model as due to a higher-order term. Second, we measure features on the subatomic scale with certain tips at low tip-sample distance, which are most likely caused by elastic interactions between the tip and the surface.
\end{abstract}

DOI: 10.1103/PhysRevB.68.205327

PACS number(s): 73.61.Ey, 68.37.Ef, 73.20.-r, 71.10.-w

\section{INTRODUCTION}

There is a continuing interest in the atomic and electronic structures of III-V surfaces, which is related to the technological importance of these materials. In particular, the defect-free (110) surface has been investigated intensively. Angle resolved photoemission spectroscopy and numerical calculations on $\mathrm{AlAs},{ }^{1} \mathrm{AlSb},{ }^{2} \mathrm{GaAs},{ }^{3-7} \mathrm{GaP}^{7-12} \mathrm{GaSb},{ }^{2}$ InAs, ${ }^{7,9,13-16} \mathrm{InP}^{7,10,12,17}$ and $\mathrm{InSb}^{9,18}$ are reported. However, detailed studies of the electronic structure on the atomic scale are rare. Some scanning tunneling microscopy (STM) and scanning force microscopy measurements are available (e.g., Refs. 19-24), but only in very few papers theoretical local electronic structure calculations and STM images are directly compared. ${ }^{10,12,25}$

The latter studies are all focussed on the large band-gap semiconductors GaAs, GaP, and InP, which exhibit an important difference to small band-gap III-V semiconductors as InAs and InSb. In the first species, surface states are dominating the STM images at all voltages, ${ }^{10}$ while in the second species the density of states (DOS) at small positive and negative energy does not contain any surface state, which allows, e.g., to probe bulk properties by STM. ${ }^{26,27}$

On the InAs(110) surface, scanning probe microscopy studies with atomic resolution exist, ${ }^{19,22,28-31}$ but a detailed comparison between STM results and electronic structure calculations is missing. Only for cross-sectional STM results on InAs/GaSb heterostructures a comparison with calculations exists, ${ }^{32}$ but this comparison is restricted to the identification of the different possible InAs/GaSb interfaces.

Here, we perform a comparison between STM results of the clean $\operatorname{InAs}(110)$ surface and calculations within the localdensity approximation of the density-functional theory. This comparison is performed using the Tersoff-Hamann model. ${ }^{33,34}$ We found, that the anion (As) is visible not only at negative sample bias as in the large band-gap materials, but also at positive bias voltages up to about $1 \mathrm{~V}$, where the indium dangling bond (In-DB) state sets in. The reason is that the local DOS (LDOS) of the parabolic bulk conduction band $(\mathrm{CB})$ has a surface appearance very similar to the arsenic dangling bond (As-DB).

In addition, we show two anomalous types of STM images on $\operatorname{InAs}(110)$. First, we find atomic resolution at $-250 \mathrm{meV}$ in both topography and the simultaneously recorded $d I / d V$ images, although this voltage corresponds to an energy in the middle of the band gap. Surprisingly this atomic resolution can be explained within the TersoffHamann model, if one takes additional terms into account. The second anomalous imaging occurs with certain tips, if the tunneling current is increased. Sharp subatomic structures are observed in the topography, which are attributed to elastic interactions between the tip and the surface atoms.

\section{CAlCulations}

Electronic structure calculations are performed using the density-functional theory (DFT) (Ref. 35) in the localdensity approximation (LDA). ${ }^{36}$ The Kohn-Sham equations are solved using the full-potential linearized augmented plane-wave method ${ }^{37}$ implemented in the FLEUR computer code. $^{38}$ To simulate the InAs(110) surface, we use a ninelayer InAs slab consisting of muffin-tin (MT) spheres around each In and As atom with radii of $1.06 \AA$ each and an interstitial region in between. The plane-wave cutoff for the basis functions is set to $k_{\max }=4.0$ a.u. ${ }^{-1}$. This corresponds to about 460 basis functions per InAs unit cell. In the MT's we use spherical harmonics with angular momentum up to $l$ $=9$. Self-consistent iterations to determine the atomic relaxation are performed with $16 k$ points in the irreducible wedge of the two-dimensional Brillouin zone. The electronic properties (LDOS) are determined with $225 k$ points. We proceed as follows. First, the theoretical lattice constant is calculated in a bulk unit cell to be $6.03 \AA$ in accordance with experimental results. ${ }^{39}$ After that, the (110) surface is introduced using the nine-layer slab and relaxed using the energy mini- 
malization method. This results in relaxation values as shown in Fig. 1(a) which are in agreement with previous calculations ${ }^{7,12,39}$ and with measurements. ${ }^{39,40}$ Finally, we analyze the electronic structure of the slab using the increased number of $k$ points.

In order to compare the STM constant current mode $(\mathrm{CCM})$ and $d I / d V$ images with theory, we need to simulate these images from the electronic structure. Although the Tersoff-Hamann model ${ }^{33,34}$ can usually not be applied to interpret atomic resolution data on metals, ${ }^{41}$ it is successful in the interpretation of atomic-resolution data on III-V semiconductors. ${ }^{10}$ This is probably due to the large atomic distances in the semiconductors and related to the less extreme tunneling conditions, where atomic resolution is obtained. Therefore, we neglect more complicated theories that take tip-sample interactions into account (e.g., Ref. 42). Within the Tersoff-Hamann model, the experimentally determined tunneling current is related to the LDOS of the sample $\rho_{s}(E, x, y)$ at the lateral position $(x, y)$ of the tip by

$$
I_{t} \propto \int_{0}^{e V} \rho_{t}(e V-E) \rho_{s}(E, x, y) T(E, V, z) d E
$$

with $\rho_{t}$ being the LDOS of the tip, $T(E, V, z)=e^{-2 \kappa(E, e V) z}$ the transmission coefficient, and $\kappa$ the decay rate. The $d I / d V$ signal is consequently related to the LDOS by

$$
\begin{aligned}
\left(\frac{d I}{d V}\right)_{V} \propto & \rho_{t}(0) \rho_{s}(e V, x, y) T(e V, V, z) \\
& +\int_{0}^{e V} \rho_{t}(e V-E) \rho_{s}(E, x, y) \frac{d T(E, V, z)}{d V} d E \\
& +\int_{0}^{e V} \frac{d \rho_{t}(e V-E)}{d V} \rho_{s}(E, x, y) T(E, V, z) d E .
\end{aligned}
$$

At low voltage, the second and third terms can usually be neglected. Only $\rho_{s}$ and $T$ have an $(x, y)$ dependence and $\rho_{s}(x, y) T(z(x, y))$ is identical to the $\operatorname{LDOS}(x, y)$ of the sample at the position of the tip at height $z$ above the surface. Consequently, to first order the calculated $d I / d V$ images are identical to the $\operatorname{LDOS}(x, y)$ at a height $z$ above the surface. In order to increase the signal-to-noise ratio, this $\operatorname{LDOS}(x, y)$ is integrated over an energy interval $\Delta E=60 \mathrm{meV}$ at each point $(x, y)$.

Experimental CCM images are compared with $\operatorname{LDOS}(x, y, z)$ images integrated over larger energy intervals assuming that $\rho_{t}$ does not depend on energy [Eq. (1)]. Partly the integration starts at $0 \mathrm{meV}$ and goes to the energy corresponding to the applied voltage, but partly the integral interval is shifted in energy to take tip-induced band bending into account. ${ }^{43,44}$ We checked that using the more complicated calculation of height profiles corresponding to constant LDOS contours, which would exactly correspond to CCM images, differs only slightly from the integrated LDOS images at constant height. This is shown in Fig. 5(c) and 5(e). Figure 5(c) shows the LDOS at a constant height of $3.5 \AA$, while a calculated CCM image with constant LDOS at about

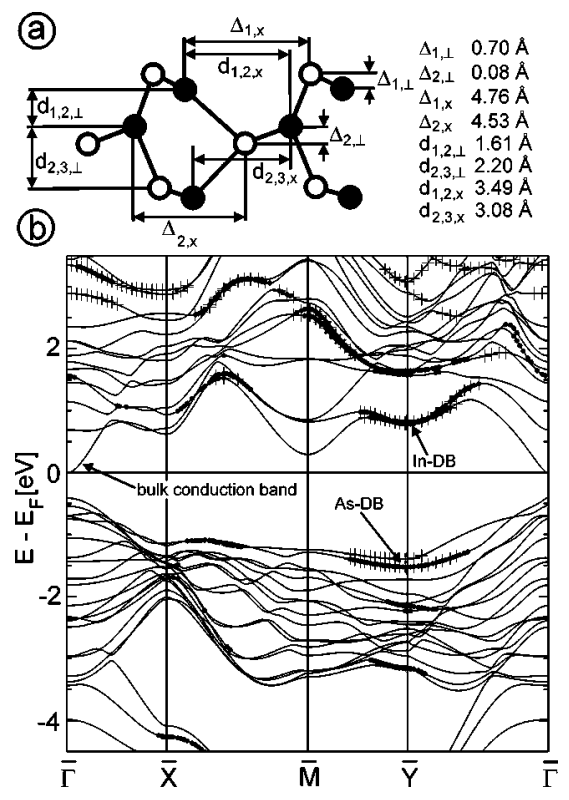

FIG. 1. (a) Relaxation of the InAs(110) surface with indicated atomic distances (black dots, In; white dots, As). The calculated values of the relaxed distances are listed on the right. (b) $\operatorname{InAs}(110)$ band structure. Large symbols mark states that lie more than $80 \%$ in the upper two layers, pluses $(+)$ mark states with more than $15 \%$ probability in the vacuum. The states corresponding to the dangling bonds of the In and As atoms as well as the bulk conduction band at $\bar{\Gamma}$ are marked.

3.5 $\AA$ is visible in Fig. 5(e). Line sections over the As atoms along the [110] direction are plotted in Figs. 5(f). Since both images and the line sections are nearly identical, we omit the more complicated procedure, and use the integrated LDOS images for comparison with STM CCM images.

\section{EXPERIMENTS}

Scanning-tunneling-microscopy measurements were performed in a low-temperature ultra high vacuum system described elsewhere. ${ }^{45}$ It operates at $T=6 \mathrm{~K}$. The InAs samples are cleaved in situ at a pressure $\leqslant 10^{-8} \mathrm{~Pa}$ and transferred into the cryostat within 5 min resulting in a clean (110) surface with an adsorbate density of about $10^{-7} / \AA^{2}$. The samples are $n$-doped $\left(N_{\mathrm{D}}=1.1 \times 10^{16} \mathrm{~cm}^{-3}\right)$, which leads to a Fermi energy about $10 \mathrm{meV}$ above the conduction bandminimum (CBM). The $\mathrm{W}$ and $\mathrm{Pt} / \mathrm{Ir}$ tips are prepared in situ by field emission and voltage pulses on a $\mathrm{W}(110)$ surface until stable imaging is obtained. All topography measurements are performed in CCM and the $d I / d V$ images are recorded simultaneously to the CCM images by lock-in technique. A modulation voltage $V_{\text {mod }}$ at frequency $f=1.6 \mathrm{kHz}$ is used.

In order to compare measured $d I / d V$ images with calculated LDOS images, we normalize the $d I / d V$ images according to the method described in Ref. 26. The method transforms $d I / d V$ images recorded in CCM into $d I / d V$ images recorded at constant height. Therefore, one first measures $I(z)$ curves, which are assumed to be proportional to the transmission coefficient $T(z)=e^{-2 \kappa z}$ with $2 \kappa$ being the at- 


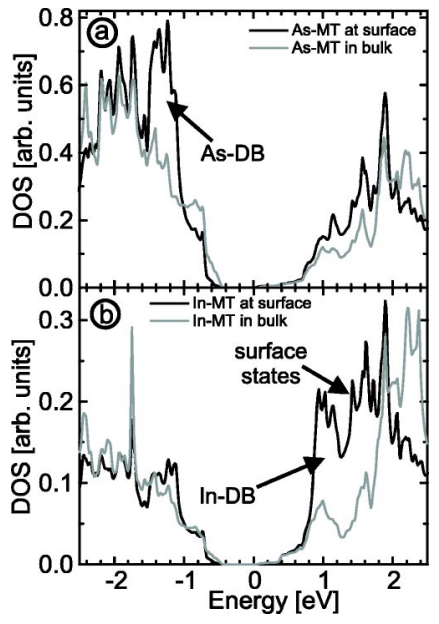

FIG. 2. Local density of states spatially integrated over MT regions. Black lines correspond to MT's directly at the surface and gray lines to atoms in the middle of the slab. (a) As-MT's, (b) In-MT's. Regions corresponding to three different surface states are marked and are discussed in the text.

tenuation coefficient. Then the CCM image $z(x, y)$ is used to solve

$$
\left[\frac{d I}{d V}(x, y)\right]_{\text {norm }}=\frac{d I / d V(x, y)}{e^{-2 \kappa z(x, y)}},
$$

which is proportional to the $d I / d V(x, y)$ at constant height.

The $d I / d V$ curves are recorded with the same lock-in technique as the $d I / d V$ images, but the tip-surface distance is kept constant after stabilizing the tip-surface distance at voltage $V_{\text {stab }}$ and current $I_{\text {stab }}$.

\section{THEORETICAL RESULTS}

The band structure of the relaxed InAs slab projected on the (110) surface is shown in Fig. 1(b). Its general structure is the same as found in earlier calculations. ${ }^{7,9}$ Larger points indicate states lying with more than $80 \%$ in the upper two layers of the film, while pluses $(+)$ show states with more than $15 \%$ probability in the vacuum, i.e., outside the MT's of the upper layer. These states are basically attributed to surface states. However, pluses at high energies, which are not coincident with larger points, might also be due to the reduced potential barrier height at such energies and might therefore belong to bulk states. Unambiguous surface states are found at different energies around the $\bar{Y}$ point, between the $\bar{X}$ and the $\bar{M}$ point as well as between the $\bar{Y}$ and the $\bar{M}$ point, but not around the $\bar{\Gamma}$ point. Note that our experiments on low-dimensional electron systems are all performed in the marked, nearly parabolic band at positive energies around the $\bar{\Gamma}$ point and are, thus, related to electrons within an InAs bulk band. ${ }^{26,27,43,46-48}$

The density of states within the MT's corresponding to the different atoms is plotted in Fig. 2. In Fig. 2(a) the DOS of a surface As-MT is compared with that of a bulk As-MT, while in Fig. 2(b) the DOS of a surface and bulk In-MT are compared. At some energies the surface DOS is highly en- hanced with respect to the bulk DOS, again indicating the surface states, but now related to particular atoms. For example, the additional surface DOS at $-1.3 \mathrm{eV}$ in the As-MT is assigned to the occupied As-DB, because (I) it is concentrated at the As atom as visible in Fig. 2(a), (II) it has a tilted $p_{z}$-like structure as can be seen from the cut through the corresponding LDOS in Fig. 3(a) and (III) it reaches deeply into the vacuum, as visible in Fig. 1 as well as in Fig. 3(a). Obviously it is centered around the $\bar{Y}$ point as can be seen in Fig. 1(b).

The In-DB can be identified in the same way by looking at Fig. 2(b) and the cross sections of the LDOS in Figs. $3(\mathrm{~d})-3(\mathrm{f})$. It is located at $0.8-1.2 \mathrm{eV}$ above the CBM, has the typical tilted $p_{z}$ structure at the In atom [Fig. 3(f)], and is centered around the $\bar{Y}$ point as well [Fig. 1(b)]. Another surface state concentrated between the $\bar{Y}$ and $\bar{M}$ points around $1.5 \mathrm{eV}$ above the CBM is identified in Figs. 3(g) and 3(h) to be of an $s p$-like type on both the As and the In atoms.

LDOS sections at $2 \AA$ above the surface for each of these three surface states are shown in Fig. 4. Evidently, only the As atom appears at energies corresponding to the As-DB, the In atom is dominant at the In-DB, and both atoms are visible with similar intensity at the third surface state. Note that the As atom is also slightly visible at In-DB energies, which we attribute to the $s p$-like configuration around the As as visible in Fig. 3(d).

The fact that all surface states are well away from the band edges leads to a peculiarity in STM images. The anion As dominates at small positive sample bias, where only the bulk CB marked in Fig. 1(b) can contribute. This is contrary to large-gap materials, where only the cation ( $\mathrm{In}, \mathrm{Ga}$ ) is measured at positive bias. ${ }^{10,12,25}$ The arsenic appearance on InAs can already be inferred from Figs. 3(b) and 3(c), where the LDOS belonging to the parabolic bulk $\mathrm{CB}$ close to $\bar{\Gamma}$ is shown. It has a dominating surface appearance as the As-DB, reaching significantly into vacuum. We will discuss this in more detail below.

\section{COMPARISON WITH STM IMAGES}

Figure 5 shows measured CCM images at small positive voltage in comparison with calculated topography. The measured images are recorded with the same tip, but at different current and voltage. The decreased voltage and increased tunneling current in Fig. 5(b) leads to a smaller tip-surface distance than in Fig. 5(a). The difference can be estimated from the measured exponential decay of the tunneling current $I=I_{0} e^{-2 \kappa \Delta z}$ with a measured value of $-2 \kappa$ $=-14.5 \mathrm{~nm}^{-1} .{ }^{49}$ This tells us that the height difference between the two measurements in Figs. 5(a) and 5(b) is $\Delta z$ $\approx 3 \AA$ A. Consequently, we compare the measured images with calculated topography images at different height, i.e., at 3.5 $\AA$ and $1 \AA$, respectively. The distances are much smaller than typical tunneling distances, but this has already been noticed by Engels et al. ${ }^{10}$ and has been attributed to influences of higher orbital tip states. The calculated topography images are shown in Figs. 5(c) and 5(d). The LDOS integral is taken over the energy range $100-300 \mathrm{meV}$, which includes the 


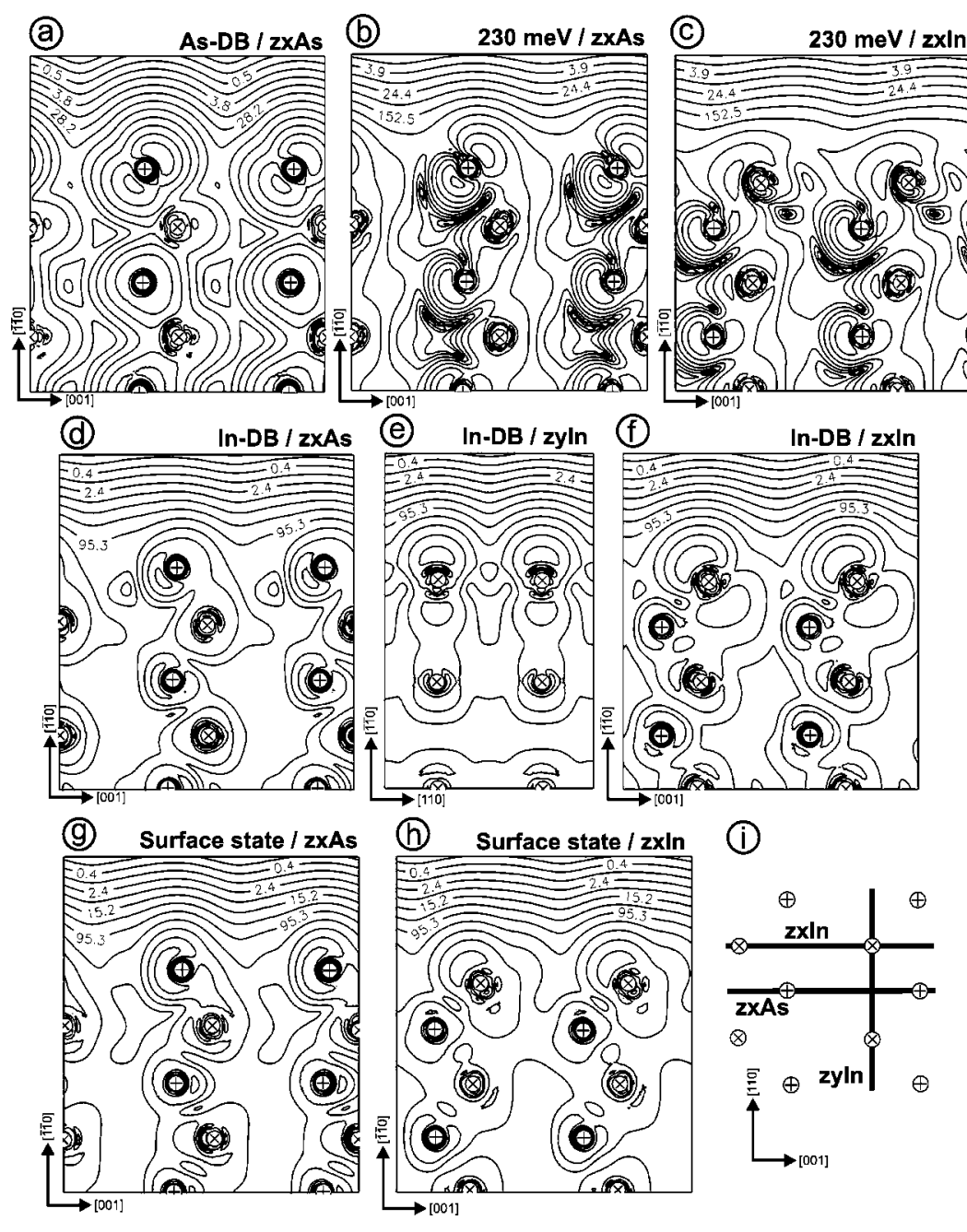

FIG. 3. LDOS cross sections taken perpendicular to the (110) surface in a way indicated by the labels, e.g., the label zxAs means that the cut is made through the As atoms in the zx-plane as defined in (i). The positions of In $(\otimes)$ and As $(\oplus)$ are marked. The numbers at the contour lines are in arbitrary units, but the same arbitrary units are used for images within one surface state. The Fermi energy is positioned at the CBM. (a) Integrated LDOS from -1500 to $-1100 \mathrm{meV}$ (As-DB). (b), (c) Integrated LDOS from 190 to $310 \mathrm{meV}$ (bulk $\mathrm{CB}$ at $\bar{\Gamma}$ ). (d)-(f) Integrated LDOS from 870 to $1200 \mathrm{meV}$ (In-DB). (g), (h) Integrated LDOS from 1450 to $1800 \mathrm{meV}$ (surface state). (i) Sketch of the surface with crosssection planes used in (a)-(h) marked.

100-meV band bending deduced straightforwardly from $d I / d V$ curves exhibiting states of the tip-induced quantum dot. $^{43}$ The agreement between measurement and calculation is excellent. Since the calculated topography at $3.5 \AA$ shows
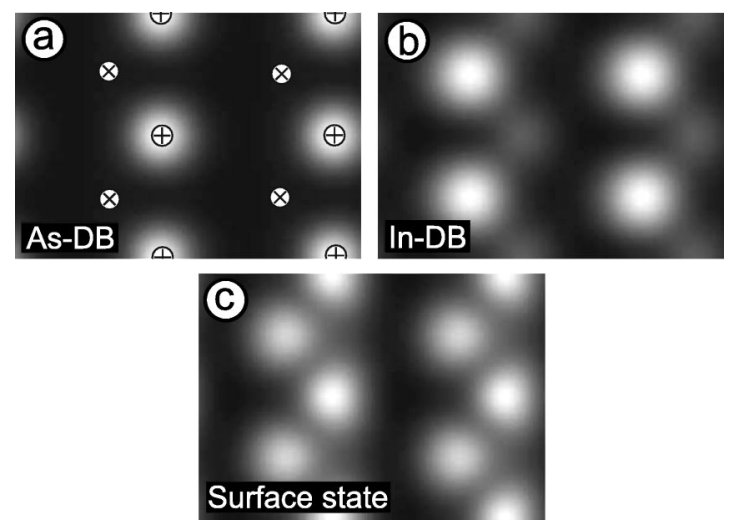

FIG. 4. LDOS distribution at $2 \AA$ above the surface. (a) Integrated LDOS from -1500 to $-1100 \mathrm{meV}$ (As-DB). (b) Integrated LDOS from 870 to $1200 \mathrm{meV}$ (In-DB). (c) Integrated LDOS from 1450 to $1800 \mathrm{meV}$ (surface state). Energies are given with respect to the CBM. The positions of the In $(\otimes)$ and As $(\oplus)$ atoms are marked in (a).
As atoms, while only closer to the surface also In atoms are visible, we attribute the intensity in the measured images accordingly to the two atoms. So we can conclude that the As atoms dominate STM images at small positive $V$. We find little change in the atomic appearance in experiment and calculation up to about $650 \mathrm{meV}$. From Fig. 1(b) one infers that this energy range is exactly the range of the parabolic $\mathrm{CB}$ around $\bar{\Gamma}$. Thus, we attribute the As appearance to this band in accordance with Fig. 3(b). Figures 5(c) and 5(e) show the comparison between integrated LDOS images and real CCM images as described in Sec. II.

Calculated topography images for higher energies taken at $5 \AA$ above the surface are shown in Fig. 6. In Fig. 6(b) (680 $\mathrm{meV})$, an additional intensity appears at the In atoms, leading to a zigzag topography. At this energy the In-DB starts to play a role, as visible in Fig. 1(b). In Fig. 6(c) $(1200 \mathrm{meV})$, only the In atoms are visible showing that the In-DB DOS now dominates with respect to the As DOS from the bulk CB at $\bar{\Gamma}$.

The transition to the In-DB regime can be observed more clearly in the calculated LDOS images in Figs. 6(d1)-6(i1). The LDOS images are compared with measured $d I / d V$ images, all recorded with the same tip in Figs. 6(d2)-6(i2). Again good correspondence between measurements and cal- 

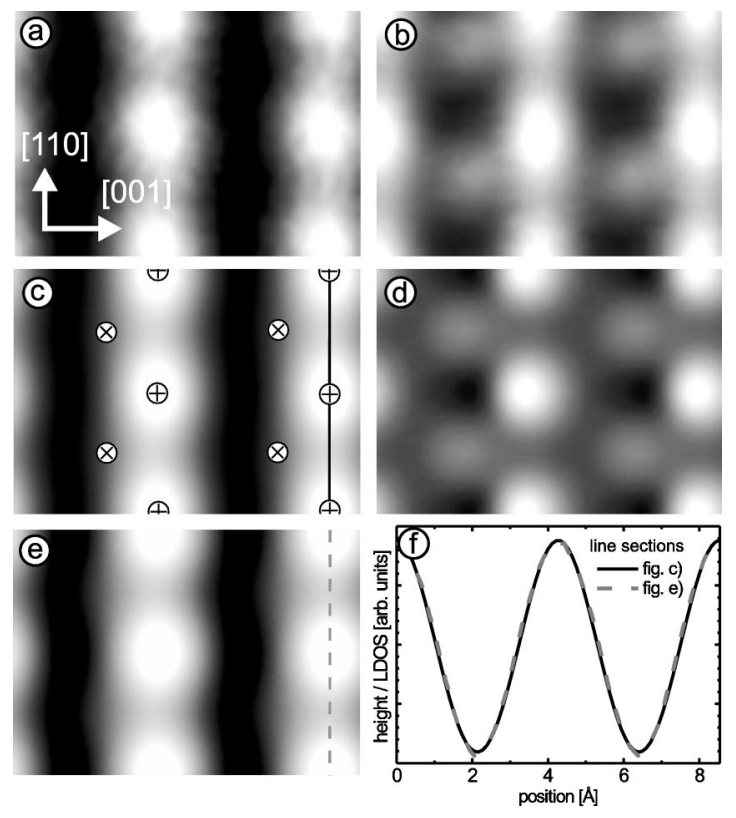

FIG. 5. Measured CCM images (a), (b) in comparison with calculations (c), (d). The tip-induced band bending is determined to be about $100 \mathrm{meV}$ (Ref. 43). (a) $V=100 \mathrm{mV}, I=200 \mathrm{pA}$. (b) $V$ $=50 \mathrm{mV}, I=1.8 \mathrm{nA}$. (c) Integrated LDOS from 100 to $300 \mathrm{meV}$ at $3.5 \AA$ above the surface. (d) Integrated LDOS from 100 to $300 \mathrm{meV}$ at $1 \AA$ above the surface. The positions of the surface In $(\otimes)$ and As $(\oplus)$ atoms are marked in (c). (e) Calculated CCM image at about $3.5 \AA$ A. (f) Line sections over the As atoms in [110] direction as marked in (c) and (e).

culations is found. However, the voltages do not correspond directly to the energies. The apparent difference of about 500 $\mathrm{meV}$, being voltage independent in the whole voltage range above $700 \mathrm{mV}$, can be attributed to the tip-induced band bending. ${ }^{43}$ A nearly constant value of $500 \mathrm{meV}$ is reasonable, since this is slightly higher than the band gap of InAs. Then, screening of holes becomes important, giving a band bending rather independent of applied voltage. A detailed discussion of this effect can be found in Ref. 44.

Next, we discuss Fig. 6(d-i) in more detail. In Fig. 6(d), the LDOS of the bulk CB at $\bar{\Gamma}$ is shown again being concentrated around the As. At an energy of $710 \mathrm{meV}$, an additional LDOS intensity is seen above the In [Fig. 6(e)]. A sharp transition into an LDOS completely centered above the In takes place within $50 \mathrm{meV}$, i.e., between Fig. 6(e) and 6(f). This is exactly at the energy where the In-DB band starts in Fig. 1(b). Between $760 \mathrm{meV}$ and $820 \mathrm{meV}$, the LDOS above the In atoms broadens into the [001] direction. This leads to apparent atomic rows rotated by $90^{\circ}$ with respect to the previous images. Above $820 \mathrm{meV}$, the broadening of the intensity in the $[00 \overline{1}]$ direction disappears again [Fig. 6(h)] and at $1250 \mathrm{meV}$, where according to Figs. 1(b) and 2(b) the In-DB region ends, the main LDOS intensity is rotated back into the [110] direction [Fig. 6(i)] ${ }^{50}$ Note that Figs. 6(f) and 6(i), both limiting the In-DB regime, are very similar. At even higher energy the third surface state with LDOS located at the In and As atoms comes into play, but STM imaging with
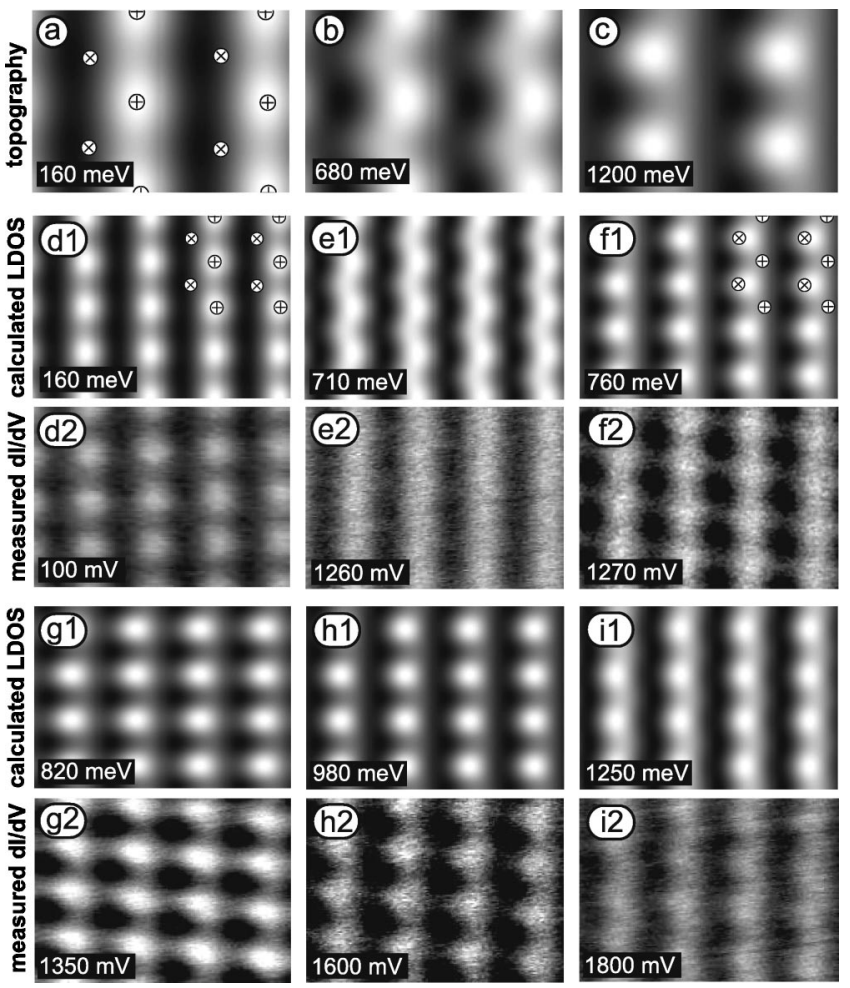

FIG. 6. (a)-(c) Calculated topography images $5 \AA$ above the surface taken at energies as indicated, with $E_{\mathrm{F}}$ at the CBM. (d1)(i1) Calculated LDOS at $5 \AA$ above the surface with marked energies given with respect to the CBM. (d2)-(i2) Measured and according to Eq. (3) normalized $d I / d V$ images taken at voltages as indicated, $I=1500 \mathrm{pA}, V_{\mathrm{mod}}=20-40 \mathrm{mV}$. The small rotation observed in the measured data is attributed to thermal drift of the sample. All measurements are recorded with the same tip. The $\otimes$ and $\oplus$ mark the positions of In and As atoms, respectively.

atomic resolution at these high voltages was unstable.

We conclude that the In-DB induces a shift from LDOS intensity from the As to the In atoms and a partial rotation of the LDOS rows from the [110] into the [001] direction. Moreover, we conclude that the measured $d I / d V$ images can largely be identified with the calculated LDOS within LDA. This confirms that the first term in Eq. (2) is dominating at moderate voltages.

\section{ATOMIC RESOLUTION IN THE BAND GAP}

In the preceding section, we successfully identified $d I / d V$ images at small positive bias with LDOS images. However, at small negative bias, where the energy corresponds to the bulk band gap, this picture has to be modified. A simplified band structure of the $n$-doped InAs(110) surface below an STM tip is shown in Fig. 7(a). It exhibits a Fermi energy at $10 \mathrm{meV}$ above the bulk CBM and a tip-induced band bending downwards by about $300 \mathrm{meV}^{43}$ Two tip-induced quantum dot states are induced in such a band bending and are drawn at -30 and $-50 \mathrm{meV}$. A $d I / d V$ curve within the same energy region is shown in Fig. 7(b). A small but nonvanishing $d I / d V$ intensity is found at voltages between $0 \mathrm{mV}$ and 


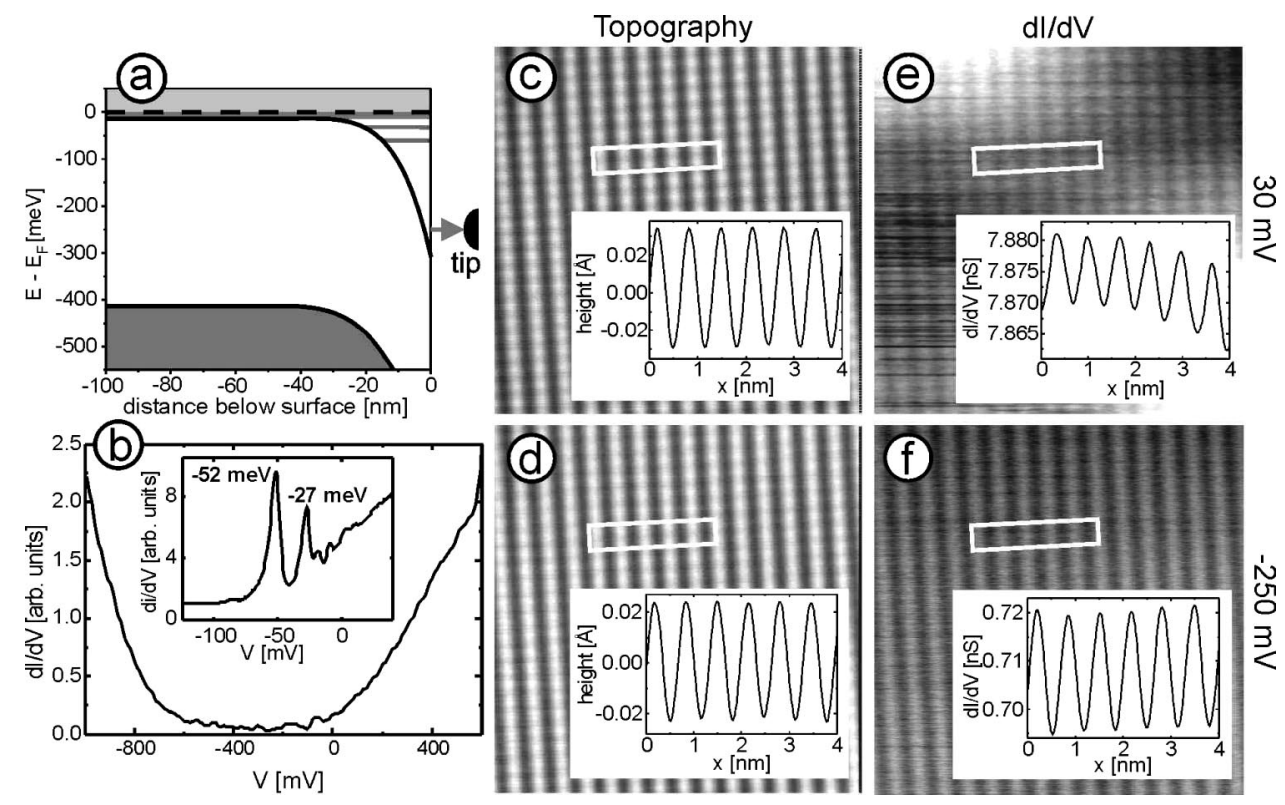

FIG. 7. (a) Sketch of the band structure of InAs including tip-induced band bending. The position of the tip indicates $V=-250 \mathrm{mV}$. (b) Spatially averaged $d I / d V(V)$ curve, $V_{\text {stab }}=600 \mathrm{mV}, I_{\text {stab }}=300 \mathrm{pA}, V_{\text {mod }}=1 \mathrm{mV}$. Inset: higher resolution $d I / d V$ curve exhibiting two quantum dot states as indicated, $V_{\text {stab }}=70 \mathrm{mV}, I_{\text {stab }}=300 \mathrm{pA}, V_{\text {mod }}=1 \mathrm{mV}$. (c), (d) CCM images, recorded simultaneously at $V=30$ and $V=-250 \mathrm{mV}$ in trace and retrace, respectively, $I=300 \mathrm{pA}$. (e), (f) $d I / d V$ images recorded simultaneously to the CCM, $V_{\text {mod }}=8 \mathrm{mV}$. Insets in (c)-(f) show line sections along the rectangles. All measurements in (b)-(f) are recorded simultaneously with the same tip.

$-600 \mathrm{mV}$. The nonzero intensity in this region, where $\rho_{s}$ $=0$, can be understood by Eq. (2): ${ }^{49}$ the $d I / d V$ signal can vanish only if all three terms in Eq. (2) are zero. But $\rho_{t}$ as well as $T(z)$ are nonzero at every voltage. Moreover, Fig. 7 (a) shows, that $\rho_{s}$ is larger than zero at the quantum dot states and between -10 and $0 \mathrm{mV}$. Therefore, the integrals in the second and third term of Eq. (2) do not vanish at negative $V$, resulting in a finite $d I / d V$ signal also at negative $V .{ }^{49}$

Moreover, the low-intensity region appears larger than the InAs band gap of $400 \mathrm{mV}$. This can be attributed to the band bending. At large negative voltage, the bands are bent downwards, leading to an additional tunnel barrier between $-400 \mathrm{mV}$ and $-700 \mathrm{meV}$. It starts at the surface and ends at the valence band onset inside the bulk [Fig. 7(a)]. Only below about $-700 \mathrm{mV}$, the valence band reaches up to the surface leading to a stronger increase in $d I / d V$.

The inset in Fig. 7(b) shows a closeup of the $d I / d V$ curve, which exhibits two quantum dot states at -52 and $-27 \mathrm{mV}$. Knowing these energies, we can estimate the band bending to be $300 \mathrm{meV}$ according to Ref. 43. This band bending is in agreement with the above interpretation. Note that this excludes any sample states between $-60 \mathrm{meV}$ and $-400 \mathrm{meV}$.

STM-CCM images recorded simultaneously at 30 and $-250 \mathrm{mV}$ in trace and retrace are shown in Fig. 7(c) and $7(\mathrm{~d})$. Atomic resolution is visible in both images, but this is not surprising, because in both cases the integral of Eq. (1) covers energy regions with states at the surface. For $-250 \mathrm{mV}$ these states are the quantum dot states and the states between the CBM and $E_{F}$. Both originate from the parabolic $\mathrm{CB}$ around $\bar{\Gamma}$ [Fig. 1(b)]. For $30 \mathrm{mV}$, these states are the states between $E_{F}$ and $30 \mathrm{meV}$ again originating from the CB at $\bar{\Gamma}$. Since the surface LDOS does not change within the parabolic band up to $\mathrm{CBM}+650 \mathrm{meV}$, i.e., between $-300 \mathrm{meV}$ and $350 \mathrm{meV}$ with respect to $E_{F}$, it is not surprising that the atomic rows at 30 and $-250 \mathrm{mV}$ appear identical and in particular, are not phase shifted (see inset).

In addition, both CCM images exhibit the same corrugation of $0.05 \AA$. This is also reasonable, since both images are recorded at the same current. Consequently, they refer to the same absolute value of integrated LDOS according to Eq. (1), which naturally should lead to the same corrugation.

Surprisingly, the $d I / d V$ images in Figs. 7(e) and 7(f) show atomic corrugation at 30 and $-250 \mathrm{mV}$, too. Note that the rows of the $d I / d V$ image at $30 \mathrm{mV}$ [Fig. 7(e)] are one third of an atomic row shifted with respect to the $d I / d V$ image at $-250 \mathrm{mV}$ [Fig. 7(f)], i.e., only the atomic rows at $-250 \mathrm{mV}$ are in phase with the rows of the two CCM images.

The insets in Figs. 7(e) and 7(f) reveal the $d I / d V$ corrugation

$$
C=\frac{(d I / d V)_{\max }-(d I / d V)_{\min }}{(d I / d V)_{\text {mean }}}
$$

to be $0.13 \%$ at $30 \mathrm{mV}$ and $3 \%$ at $-250 \mathrm{mV}$ and an absolute $d I / d V$ signal of $0.71 \mathrm{nS}$ at $-250 \mathrm{mV}$ and $7.9 \mathrm{nS}$ at $30 \mathrm{mV}$.

The latter numbers are not directly comparable because of a different tip height in the two measurements. But since we measured that the tip is $\Delta z=0.9 \AA$ closer to the surface at 30 $\mathrm{mV}$, we can calculate a height-normalized $d I / d V$ ratio of 


$$
\frac{(d I / d V)_{-250}}{(d I / d V)_{30}}=\frac{0.71}{7.9 e^{-2 \kappa \Delta z}}=0.3
$$

This ratio of $d I / d V$ values between -250 and $30 \mathrm{mV}$ can also be observed in the $d I / d V$ curve in Fig. 7(b).

The question to discuss is: what causes the atomic corrugation at $-250 \mathrm{mV}$ in the $d I / d V$ images, where $\rho_{s}=0$ ?

A first reason could be that during the scan, the tip height varies, which can induce an atomic $d I / d V$ corrugation caused by the atomic variation of $z$ and, thus, of $T(z)$ $\propto d I / d V$ [Eq. (2)]. However, the $d I / d V$ image has maxima at the same position as the corresponding CCM image, while $T(z)$ varies in antiphase with $z$. Thus, we can exclude this explanation.

Another possible reason could be the influence of states made available by the band bending. Such an explanation has been proposed for GaAs(110) by De Raad et al. ${ }^{51}$ However, the two quantum dot states induced by the band bending in the InAs case are measured to be at $-52 \mathrm{mV}$ and $-27 \mathrm{mV}$ far away from $-250 \mathrm{mV}$. Consequently, we can exclude such an explanation as well.

Finally, we could understand our corrugation by the second and third terms of Eq. (2). To estimate the contribution of these terms, we first note that $\rho_{s}(E)$ is largely constant from $-10 \mathrm{mV}$ to $30 \mathrm{mV}$ in accordance with Fig. 7(b). Moreover, we assume that the $\rho_{s}(E)$ corresponding to the two quantum dot states [inset of Fig. 7(b)] can be replaced by a constant $\bar{\rho}_{s}$ in the region from $-60 \mathrm{mV}$ to $-10 \mathrm{mV}$. The resulting $\bar{\rho}_{s}$ is approximately the same as $\rho_{s}$ measured at small positive $V$, leading to

$$
\rho_{s}= \begin{cases}0 & \text { for }-250 \mathrm{mV} \leqq V<V_{\mathrm{s}} \\ \bar{\rho}_{s} & \text { for } V_{\mathrm{s}} \leqq V \leqq 30 \mathrm{mV}\end{cases}
$$

with $V_{\mathrm{s}}=-60 \mathrm{mV}$. Finally we assume as usual that $(d I / d V)_{30}(=d I / d V$ at $30 \mathrm{mV})$ is dominated by the first term in Eq. (2), which is correct as long as $d \rho_{t} / d V$ $<\rho_{t} / 30 \mathrm{mV}^{49}$

To estimate the influence of the second term on $(d I / d V)_{-250}$, a relation between $T$ and $d T / d V$ is required. $T$ is usually expressed by the exponent $e^{-2 \kappa z}$ with $\kappa$ $=\sqrt{2 m(\phi-e V)} / \hbar$ (see, e.g., Ref. 52). With the measured barrier height, $\phi=1.3 \mathrm{eV}$, and a typical tip-sample distance of $z=5 \AA$, we obtain the expression $d T / d V \approx 0.33 / \mathrm{V} \cdot T$. Using Eq. (6) this results in

$$
\begin{aligned}
\frac{(d I / d V)_{-250}}{(d I / d V)_{30}} & =\frac{\int_{0}^{V_{s}} \rho_{t} \overline{\rho_{s}} \frac{d T}{d V} d V}{\rho_{t}(0) \rho_{s}(30) T} \\
& \approx \frac{\overline{\rho_{t}} \overline{\rho_{s}} \frac{\overline{d T}}{d V} V_{s}}{\rho_{t}(0) \overline{\rho_{s}} T} \approx 0.33 / \mathrm{V} \cdot V_{s}=0.02 .
\end{aligned}
$$

This ratio is much smaller than the measured ratio of 0.3 [Eq. (5)], so this term can also not be the origin of the $d I / d V$ signal at $-250 \mathrm{mV}$, at least if $\overline{\rho_{t}}$ is not largely different from $\rho_{t}(0)$.

The remaining possibility is the third term. However, the ratio $(d I / d V)_{-250} /(d I / d V)_{30}$ cannot be calculated, since the ratio $\left(d \rho_{t} / d V\right) / \rho_{t}$ is unknown. It is, nevertheless, possible to estimate the $\left(d \rho_{t} / d V\right) / \rho_{t}$ ratio necessary to explain a $d I / d V$ ratio of 0.3 . Using Eq. (2) again, integrating over $\left[V_{s}, 0\right]$ according to Eq. (6) and comparing with Eq. (5), we get

$$
\begin{aligned}
\frac{(d I / d V)_{-250}}{(d I / d V)_{30}} & =\frac{\int_{0}^{V_{s}} \frac{d \rho_{t}}{d V} \overline{\rho_{s}} T d V}{\rho_{t}(0) \rho_{s}(30) T}=\frac{\overline{d \rho_{t}} \overline{\rho_{s}} T V_{s}}{\rho_{t}(0) \overline{\rho_{s}} T}=\frac{\overline{d \rho_{t}}}{d V} V_{s} \\
& \approx 0.3 \Rightarrow \frac{\overline{\rho_{t}}}{d V} / \rho_{t}(0) \approx 5 \mathrm{~V}^{-1}
\end{aligned}
$$

This means that $\rho_{t}$ must change by about $30 \%$ within the region between $-60 \mathrm{mV}$ and $0 \mathrm{mV}$. This is quite possible. We conclude that the observed $d I / d V$ signal in the band gap can be understood within the Tersoff-Hamann model as due to the third term.

If this is correct, also the atomic resolution measured at $-250 \mathrm{mV}$ is explained straightforwardly. We have for $V$ $=-250 \mathrm{mV}$,

$$
\left.\frac{d I}{d V}(x, y)\right|_{V} \approx \int_{0}^{V} \frac{d \rho_{t}(e V-E)}{d V} \rho_{s}(x, y, E) T(V, E, z) d V
$$

with neither $T$ nor $\rho_{s}$ depending significantly on energy down to $V_{s}$. We get

$$
\left.\frac{d I}{d V}(x, y)\right|_{V} \approx \rho_{s}\left(x, y, E_{F}\right) e^{-2 \kappa z} \int_{0}^{V_{s}} \frac{d \rho_{t}(e V-E)}{d V} d V .
$$

Importantly, we assume that not only the absolute value of $\rho_{s}$ is energy independent, but rather that the $(x, y)$ dependence of $\rho_{s}$ does not depend on energy. However, exactly this assumption has been proven in the preceding section. Thus, $(d I / d V)_{-250}$ basically measures the $\operatorname{LDOS}(x, y)$ in the parabolic bulk conduction band, which is represented by the two quantum-dot states and the DOS in the conduction band below $E_{F}$. The necessary LDOS to explain the measured $d I / d V$ corrugation of $3 \%$ is given by a normalization according to Eq. (3) resulting in $C_{L D O S}^{-250} \approx 13 \%$.

The final question is why we observe a phase shift of the atomic rows at $30 \mathrm{mV}$. This experimental observation is less surprising, if one again applies the normalization of Eq. (3), which leads to a $d I / d V$ corrugation of $C_{L D O S}^{30} \approx 11 \%$ in phase with the one at $-250 \mathrm{mV}$. So, only a small quantitative difference between $C_{L D O S}^{-250}$ and $C_{L D O S}^{30}$ remains and we conclude that both images are caused by the LDOS of the bulk CB.

Note finally that this conclusion is independent of the major assumptions of the Tersoff-Hamann model.

(a) A $p_{z}$ character of the tip would only imply that instead of $\rho_{s}$ we should use $d \rho_{s} / d z,{ }^{53}$ which would not change the $d I / d V$ ratios in Eq. (7) or (8). An analogous argument holds for all other possible tip orbitals. 

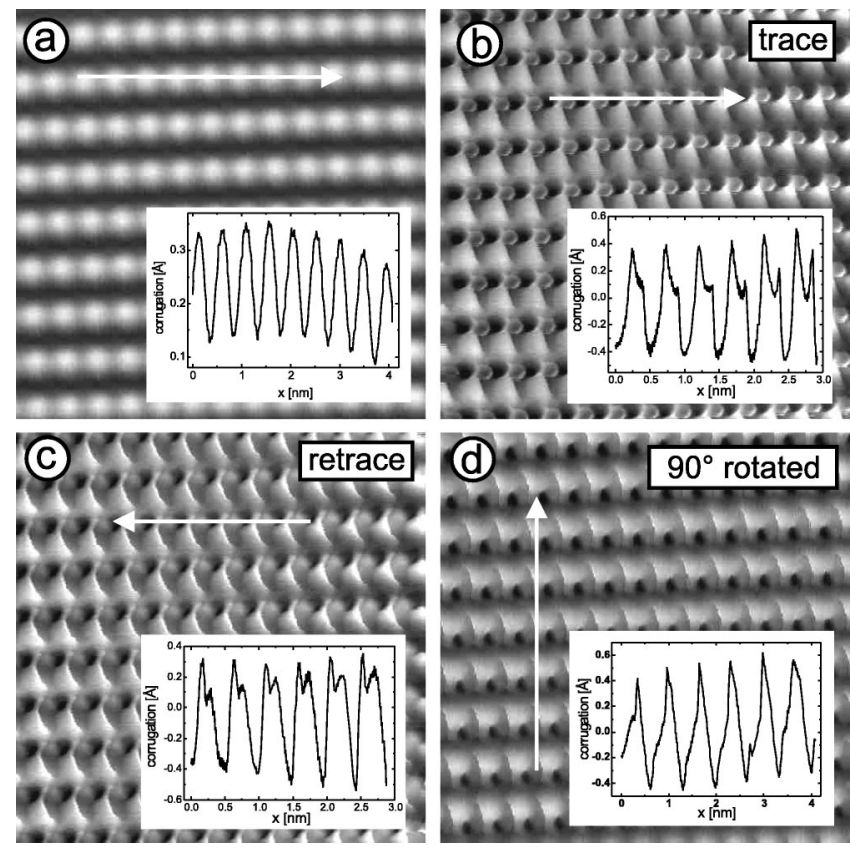

FIG. 8. CCM images all recorded with the same tip on InAs(110). (a) Standard atomic resolution. $V=50 \mathrm{mV}, \quad I$ $=450 \mathrm{pA}$. (b) $V=50 \mathrm{mV}, I=710 \mathrm{pA}$, scan direction=trace. (c) Same as in (b), but scan direction $=$ retrace. (d) Same as in (b), but scan direction rotated by $90^{\circ}$ and $V=50 \mathrm{mV}, I=530 \mathrm{pA}$. Insets show line sections along marked arrows. Arrows indicate the scan direction.

(b) $\kappa$ is not a function of lateral position, which we confirmed by recording laterally resolved $I(z)$ curves. Thus, we can indeed write $T=e^{-2 \kappa z(x, y)}$ in Eqs. (3) and (5) with constant $\kappa$.

We conclude this section by stating that atomic resolution in $d I / d V$ images corresponding to band-gap voltages is possible, if $d \rho_{t} / d V \neq 0$ and if there are any states at the surface between $E_{F}$ and the applied voltage.

\section{SMALL TIP-SAMPLE DISTANCE IMAGING}

As discussed above, atomic-resolution images recorded in CCM at small positive bias usually look like Fig. 8(a) (rotated by $90^{\circ}$ with respect to Fig. 5). They exhibit bumps at the As atoms and are straightforwardly understood in terms of the integrated LDOS. However, with certain tips, the CCM images change dramatically if the tunneling current is only slightly increased. An example is shown in Fig. 8(b). The effect occurs only with certain tips, but is then reproducible, i.e., one can switch between Figs. 8(a) and 8(b) by repeatedly increasing and decreasing the current. We checked that the tips are not multiple tips by imaging defects on the surface. Note that in the measurements shown in Fig. 8 , the transition takes place between 450 and $530 \mathrm{pA}$, i.e., it is rather sharp.

Above the transition, subatomic features are visible with an appearance strongly depending on the scan angle. The images in Figs. 8(a) and 8(b) are recorded with the same scan direction (trace), while Fig. 8(c) is recorded in opposite scan direction (retrace) and Fig. 8(d) is recorded with a scan direction rotated by $90^{\circ}$. Obviously, the features are different in all three images excluding that the unperturbed LDOS is imaged. We propose that the image mode is related to a tip-sample interaction. The fact that this interaction depends on scan direction strongly suggests that the interaction shows hysteresis.

Indeed very similar effects and images are found in contact AFM. There the elastic interaction of a tip in contact with a sample is directly measured ${ }^{54,55}$ and the sharp features on the subatomic scale are attributed to a slip-stick motion of the tip with respect to the sample. Hölscher et al. calculated that the AFM tip, while being moved over the surface, sticks in an atomic potential minimum for some time and then jumps suddenly to the next potential minimum, if the lateral force is larger than a certain threshold. ${ }^{54,55}$ Thus, one gets a continuous change of atomic force during the stick and then a sudden jump in force to the next minimum.

This model can be straightforwardly transferred to our case. First, the sharp transition in imaging between $450 \mathrm{pA}$ and $530 \mathrm{pA}$ is the equivalent of the jump to contact in AFM. However, our feedback parameter is not force but current, explaining the reversibility of the switching between the two types of imaging. In contact we still measure a resistance of $70 \mathrm{M} \Omega$, which is well above the point contact resistance of $20 \mathrm{k} \Omega$. This requires an insulating region at the tip end, probably an adsorbate at the end of the tip. The transmittivity of this insulating region depends most likely on elastic deformation. Thus, we interpret the sharp features in the CCM images as sudden jumps of the atomic contact configuration from one potential minimum to the next one changing the transmittivity. In a sense, the images can be considered as the electric response of a mechanical deformation on the nanometer scale. Although Fig. 8(d) is similar to noncontact (NC) AFM measurements performed by Schwarz et al., ${ }^{19}$ these NC-AFM measurements are the same in all scan directions, ${ }^{56}$ while our measurements are not.

\section{SUMMARY}

We performed $a b$ initio electronic structure calculations (DFT-LDA) for the InAs(110) surface. Within the calculations, we identified the surface states corresponding to the In and As dangling bonds to be located about $1 \mathrm{eV}$ away from the band edges. The LDOS within the CB is directly compared with measured $d I / d V$ images at positive voltage. We find very nice agreement. In particular, the transition from bulk states to the In-DB state has been identified in $d I / d V$ images to cause a shift of $d I / d V$ intensity from the As atom to the In atom and a rotation of the apparent atomic rows by $90^{\circ}$.

Second, we measured atomic corrugations in $d I / d V$ at voltages corresponding to the band gap, i.e., at energies where no states exist. We could also describe this contrast by the Tersoff-Hamann model as due to a higher-order term and conclude that atomic resolution is still possible, if the tip DOS is not constant as a function of energy and if there are 
any states at the surface between the applied voltage and $E_{F}$.

Finally, with some tips we switch reproducibly between normal imaging of the LDOS and a bizarre imaging showing features on a subatomic length scale. We believe that the latter is due to elastic interactions between certain STM tips and the surface.

\section{ACKNOWLEDGMENTS}

Financial support from the Graduiertenkollegs "Spektroskopie an lokalisierten atomaren Systemen" and "Physik nanostrukturierter Festkörper" of the DFG is gratefully acknowledged.
*Email address: mmorgens@ physnet.uni-hamburg.de; http:// www.nanoscience.de/group_r/stm-sts/

${ }^{1}$ H. Tütüncü and G. Srivastava, Surf. Sci. 457, 211 (2000).

${ }^{2}$ H. Tütüncü and G. Srivastava, Phys. Rev. B 59, 4925 (1999).

${ }^{3}$ J. Chelikowsky and M. Cohen, Solid State Commun. 29, 267 (1979).

${ }^{4}$ J.R. Chelikowksy and M.L. Cohen, Phys. Rev. B 13, 826 (1976).

${ }^{5}$ J.R. Chelikowsky and M.L. Cohen, Phys. Rev. B 20, 4150 (1979).

${ }^{6}$ D. Chadi, Phys. Rev. B 18, 1800 (1978).

${ }^{7}$ J.L. Alves, J. Hebenstreit, and M. Scheffler, Phys. Rev. B 44, 6188 (1991).

${ }^{8}$ F. Manghi, C. Bertoni, C. Calandra, and E. Molinari, Phys. Rev. B 24, 6029 (1981).

${ }^{9}$ R.P. Beres, R.E. Allen, and J.D. Dow, Phys. Rev. B 26, 5702 (1982).

${ }^{10}$ B. Engels, P. Richard, K. Schroeder, S. Blügel, P. Ebert, and K. Urban, Phys. Rev. B 58, 7799 (1998).

${ }^{11}$ S. Jenkins, G. Srivastava, and J. Inkson, Surf. Sci. 331-333, 1238 (1995).

${ }^{12}$ B. Engels, Ph.D. thesis, Reinisch-Westfälische Technische Hochschule, Aachen, Germany, 1995.

${ }^{13}$ C. Andersson, J. Andersen, P. Persson, and U. Karlsson, Phys. Rev. B 47, 2427 (1993).

${ }^{14}$ W. Drube, D. Straub, and F. Himpsel, Phys. Rev. B 35, 5563 (1987).

${ }^{15}$ D. Swanston, A. McLean, D. McIlroy, D. Heskett, R. Ludeke, H. Munekata, M. Prietsch, and N. DiNardo, Surf. Sci. 312, 361 (1994).

${ }^{16}$ C. Andersson, J. Andersen, P. Persson, and U. Karlsson, Surf. Sci. 398, 395 (1998).

${ }^{17}$ L. Sorba, V. Hinkel, H. Middelmann, and K. Horn, Phys. Rev. B 36, 8075 (1987).

${ }^{18}$ H. Tütüncü, M. Çakmak, and G. Srivastava, Appl. Surf. Sci. 123, 146 (1998).

${ }^{19}$ A. Schwarz, W. Allers, U. Schwarz, and R. Wiesendanger, Phys. Rev. B 61, 2837 (2000).

${ }^{20}$ L. Whitman, J.A. Stroscio, R. Dragoset, and R. Celotta, Phys. Rev. B 42, 7288 (1990).

${ }^{21}$ R. Feenstra, J.A. Stroscio, J. Tersoff, and A. Fein, Phys. Rev. Lett. 58, 1192 (1987).

${ }^{22}$ A. Schwarz, W. Allers, U. Schwarz, and R. Wiesendanger, Appl. Surf. Sci. 140, 293 (1999).

${ }^{23}$ P. Ebert, G. Cox, U. Poppe, and K. Urban, Surf. Sci. 271, 587 (1992).

${ }^{24}$ G. de Raad, D. Bruls, P. Koenraad, and J. Wolter, Phys. Rev. B 66, 195306 (2002)

${ }^{25}$ P. Ebert, B. Engels, P. Richard, K. Schroeder, S. Blügel, C. Domke, M. Heinrich, and K. Urban, Phys. Rev. Lett. 77, 2997 (1996).

${ }^{26}$ C. Wittneven, R. Dombrowski, M. Morgenstern, and R. Wiesen- danger, Phys. Rev. Lett. 81, 5616 (1998).

${ }^{27}$ D. Haude, M. Morgenstern, I. Meinel, and R. Wiesendanger, Phys. Rev. Lett. 86, 1582 (2001).

${ }^{28}$ Y. Liang, W.E. Packard, J.D. Dow, H. Ho, and G. Lapeyre, Phys. Rev. B 48, 11942 (1993).

${ }^{29}$ N. Maslova, S. Oreshkin, V. Panov, S. Savinov, A. Depuydt, and C. van Haesendonck, JETP Lett. 67, 146 (1998).

${ }^{30}$ A. Depuydt, N. Maslova, V. Panov, V. Rakov, S.V. Savinov, and C. van Haesendonck, Appl. Phys. A: Mater. Sci. Process. 66, S171 (1998).

${ }^{31}$ J. Harper, M. Weimer, D. Zhang, C.-H. Lin, and S. Pei, Appl. Phys. Lett. 73, 2805 (1998).

${ }^{32}$ S. Kim, S. Erwin, B. Nosho, and L. Whitman, Phys. Rev. B 67, 121306 (2003).

${ }^{33}$ J. Tersoff and D. Hamann, Phys. Rev. Lett. 50, 1998 (1983).

${ }^{34}$ J. Tersoff and D. Hamann, Phys. Rev. B 31, 805 (1985).

${ }^{35}$ P. Hohenberg and W. Kohn, Phys. Rev. 136, B864 (1964).

${ }^{36}$ J. Perdew and Y. Wang, Phys. Rev. B 45, 13244 (1992).

${ }^{37}$ E. Wimmer, H. Krakauer, M. Weinert, and A. Freeman, Phys. Rev. B 24, 864 (1981).

${ }^{38} \mathrm{http}: / / w w w . f l a p w . d e$

${ }^{39}$ C. Mailhiot, C. Duke, and D. Chadi, Surf. Sci. 149, 366 (1985).

${ }^{40}$ C. Duke, A. Paton, A. Kahn, and C. Bonapace, Phys. Rev. B 27, 6189 (1983).

${ }^{41}$ J. Wintterlin, J. Wiechers, H. Brune, T. Gritsch, H. Höfer, and R. Behm, Phys. Rev. Lett. 62, 59 (1988).

${ }^{42}$ D. Drakova, Rep. Prog. Phys. 64, 205 (2001).

${ }^{43}$ R. Dombrowski, C. Steinebach, M. Morgenstern, and R. Wiesendanger, Phys. Rev. B 59, 8043 (1999).

${ }^{44}$ R. Feenstra, Phys. Rev. B 50, 4561 (1994).

${ }^{45}$ C. Wittneven, R. Dombrowski, S. Pan, and R. Wiesendanger, Rev. Sci. Instrum. 68, 3806 (1997).

${ }^{46}$ M. Morgenstern, D. Haude, V. Gudmundsson, C. Wittneven, R. Dombrowski, and R. Wiesendanger, Phys. Rev. B 62, 7257 (2000).

${ }^{47}$ M. Morgenstern, J. Klijn, C. Meyer, M. Getzlaff, R. Adelung, R. Römer, K. Rossnagel, L. Kipp, M. Skibowski, and R. Wiesendanger, Phys. Rev. Lett. 89, 136806 (2002).

${ }^{48}$ M. Morgenstern, C. Wittneven, R. Dombrowski, and R. Wiesendanger, Phys. Rev. Lett. 84, 5588 (2000).

${ }^{49}$ M. Morgenstern, D. Haude, V. Gudmundsson, C. Wittneven, R. Dombrowski, C. Steinebach, and R. Wiesendanger, J. Electron Spectrosc. Relat. Phenom. 109, 127 (2000).

${ }^{50}$ On large band-gap semiconductors, only the rotation back into the [110] direction has been observed, since the cation dangling bond starts directly at the band edge (Ref. 10 and Refs. 23-25)

${ }^{51}$ G. de Raad, D. Bruls, P. Koenraad, and J. Wolter, Phys. Rev. B 64, 075314 (2001). 
${ }^{52} \mathrm{R}$. Wiesendanger, Scanning Probe Microscopy and Spectroscopy. Methods and Applications (Cambridge University Press, Cambridge, England, 1994).

${ }^{53}$ C. J. Chen, Introduction to Scanning Tunneling Microscopy (Oxford University Press, New York, 1993).
${ }^{54}$ H. Hölscher, U. Schwarz, O. Zwörner, and R. Wiesendanger, Z. Phys. B: Condens. Matter 104, 295 (1997).

${ }^{55}$ H. Hölscher, W. Raberg, U. Schwarz, A. Hasbach, K. Wandelt, and R. Wiesendanger, Phys. Rev. B 59, 1661 (1999).

${ }^{56}$ A. Schwarz (private communication). 